\title{
Thermal Excitation Control over Photon Emission Rate of CdSe Nanocrystals
}

\author{
Benjamin T. Diroll ${ }^{1}$ and Richard D. Schaller ${ }^{1,2^{*}}$ \\ ${ }^{1}$ Center for Nanoscale Materials, Argonne National Laboratory, Lemont, IL 60439 \\ ${ }^{2}$ Department of Chemistry, Northwestern University, Evanston, IL 60208 \\ *schaller@anl.gov; schaller@northwestern.edu
}




\section{Experimetnal Details}

Materials: Cadmium acetylacetonate (98\%, Aldrich), selenium (99.99\%, powder, 100 mesh, Aldrich), 1-octadecene (90\%, technical grade, Aldrich), oleic acid (90\%, technical grade, Aldrich), cadmium nitrate $(97 \%$, Aldrich), sodium myristate ( $>98 \%$, Aldrich), and oleylamine $(70 \%$, Aldrich) were sourced from commercial suppliers and used as received. Other solvents used were sourced from commercial suppliers and were ACS grade or higher.

Synthesis of Quantum Dots (QDs). CdSe QDs in the zinc blende phase were prepared via modifications of literature recipes. ${ }^{1} 2.3 \mathrm{~nm}$ and $2.6 \mathrm{~nm}$ CdSe were prepared by combining $310 \mathrm{mg}$ cadmium acetylacetonate, $350 \mu \mathrm{L}$ of oleic acid, and $30 \mathrm{~mL}$ of octadecene were heated under vacuum to $120{ }^{\circ} \mathrm{C}$ and held for 1 hour. The reaction vessel was then put under nitrogen and $40 \mathrm{mg}$ selenium powder was added by counterflow addition. The vessel was pumped out with vacuum at 10 more minutes, then heated under nitrogen to $225^{\circ} \mathrm{C}$ for the smallest NCs and $240^{\circ} \mathrm{C}$ for all others. For the $2.3 \mathrm{~nm} \mathrm{NCs}$, once the reaction reached $225^{\circ} \mathrm{C}$, it was held for 1 minute, then $1 \mathrm{~mL}$ of oleylamine, $1 \mathrm{~mL}$ oleic acid, and $4 \mathrm{~mL}$ of octadecene was rapidly injected and the vessel was cooled. For $2.6 \mathrm{~nm}$ NCs, upon reaching $240^{\circ} \mathrm{C}$, after 3 minute, $4 \mathrm{~mL}$ of octadecene combined with $1 \mathrm{~mL}$ oleylamine and $1 \mathrm{~mL}$ oleic acid was rapidly injected. The reaction was allowed to proceed for 4 more minutes, then cooled to room temperature. For $3.8 \mathrm{~nm}$ and $5.9 \mathrm{~nm} \mathrm{CdSe} \mathrm{NCs,} 275 \mathrm{mg}$ cadmium myristate prepared by precipitation using cadmium nitrate and sodium myristate dissolved in methanol was heated with $25 \mathrm{~mL}$ of octadecene and $40 \mathrm{mg}$ Se powder to $100^{\circ} \mathrm{C}$ under vacuum and held $1 \mathrm{hr}$. Then, under nitrogen, the reaction pots were heated to $240^{\circ} \mathrm{C}$. After 3 minutes at $240{ }^{\circ} \mathrm{C}$, a dropwise injection mixture of $1 \mathrm{~mL}$ oleic acid, $1 \mathrm{~mL}$ oleylamine, and $4 \mathrm{~mL}$ octadecene was started that continued for 6 minutes. For $3.8 \mathrm{~nm} \mathrm{NCs}$, the reaction was held 30 minutes after the injection, then cooled to room temperature. For $5.9 \mathrm{~nm} \mathrm{NCs,} \mathrm{the} \mathrm{reaction} \mathrm{was}$ 
held 30 minutes at $240^{\circ} \mathrm{C}$ and then heated to $280^{\circ} \mathrm{C}$. At $280^{\circ} \mathrm{C}$, a dropwise injection at 0.2 $\mathrm{mL} /$ minute of $18 \mathrm{~mL} 0.1 \mathrm{M}$ selenium dissolved in octadecene (at $300^{\circ} \mathrm{C}$ under nitrogen) and $3 \mathrm{~mL}$ of $0.5 \mathrm{M}$ cadmium oleate in oleic acid (prepared by dissolving cadmium oxide in oleic acid at $280^{\circ}$ C). After the injection was finished, the reaction pot was cooled to room temperature. All samples were isolated by three precipitations with isopropanol to remove excess unbound ligand.

Structural Characterization. X-ray diffraction measurements shown in Figure S1 were performed to confirm the cubic zinc blende phase of all samples. Measurements were performed using a Bruker D2 phaser instrument on drop-cast films on mis-cut silicon for low background. TEM measurements were performed using a JEOL-2100F microscope. Micrographs of the samples are shown in Figure S2.

Fourier Transform Infrared (FT-IR) Spectroscopy. FT-IR measurements were performed at room temperature on dried samples on sapphire. Measurements were performed on a Nicolette 6700 FTIR. Room temperature FT-IR absorption spectra in the pump pulse window are shown in Figure S3. Previous measurements of ligand absorption have also shown very little change in extinction at lower temperatures. ${ }^{2}$

Infrared Pump, Electronic Probe (IPEP) spectroscopy. Similar to previous reports, ${ }^{3,4}$ an amplified SpectraPhysics Ti: Sapphire laser operating at $2 \mathrm{kHz}$ was split into two beam to generate the pump and probe beams for the transient absorption (TA) experiments. The pump beam was generated using an optical parametric amplifier and tuned to $3460 \mathrm{~nm}$ on resonance with the $\mathrm{C}-\mathrm{H}$ stretching vibrations of hydrocarbon ligands. This beam was chopped at $1 \mathrm{kHz}$ frequency. The second beam was used to generate a visible white light by focusing through a sapphire plate to generate a supercontinuum. Data collection was performed using Ultrafast Systems detectors and Helios 
software. Total absorbed IR fluence of the $300 \mu \mathrm{m}$ spot was estimated by measuring the power of the IR beam through the measured sample spot compared to the sapphire plate without the sample. The spectral resolution of the TA experiments was $c a .13 \mathrm{~nm}$ as configured.

Temperature-Dependent Absorption Calibration. Visible spectral range, temperature-dependent absorption measurements of $\mathrm{NC}$ films on sapphire were performed using a focused white light source $(\sim 2 \mathrm{~mm})$ directed into a cryostat, collected and fiber-coupled to an OceanOptics spectrometer. To obtain a calibration of the expected change in absorption for a given change in temperature at $5 \mathrm{~K}$ in order to provide a temperature change estimate from TA data, absorption spectra were collected for each of the samples at $5 \mathrm{~K}, 15 \mathrm{~K}, 25 \mathrm{~K}, 35 \mathrm{~K}$, and $45 \mathrm{~K}$. The spectral resolution of this measurement was $c a .1 .5 \mathrm{~nm}$ as configured. Unlike measurements at higher temperature, the change in the absorption is not linear with $\Delta \mathrm{T}$; rather it is quadratic because temperature-dependent changes in the band gap are quadratic. ${ }^{5}$ (See Figure S4.) Additionally, although the heat capacity of the samples is not well-known at such temperatures, we note that simply using the absorbed power and bulk specific heat capacity leads to a much larger estimated temperature rise, implying that the heat transfer process into the inorganic particle has non-unity yield.

Time-resolved Photoluminescence Spectroscopy. Time-resolved PL spectroscopy was performed using a frequency-doubled Ti: sapphire output at $400 \mathrm{~nm}$ at $2 \mathrm{kHz}$ for the blue excitation source. The illumination spot was $\sim 1 \mathrm{~mm}$ in diameter and the power of the excitation was $17 \mu \mathrm{W}$ for a fluence of approximately $1 \mu \mathrm{J} / \mathrm{cm}^{2}$. The fluence was maintained at a low level to avoid multiexcitonic absorption and concomitant Auger heating processes. The emitted light was collected using focusing lenses and fiber-coupled into a streak camera (Hamamatsu) through a spectrometer. For those experiments with thermal excitation, a $3460 \mathrm{~nm}$ IR beam $(300 \mu \mathrm{m}$ 
diameter) was overlapped with the blue excitation spot and delayed with respect to the initial 400 $\mathrm{nm}$ pump excitation. Although the power of the IR excitation used for time-resolved PL experiments was twice that of IPEP experiments (which are chopped), the fluence was the same. The estimated absorbed fluence of the measurements was ca. $64 \mu \mathrm{J} / \mathrm{cm}^{2}$ for $5.9 \mathrm{~nm} \mathrm{NCs} ; 104$ $\mu \mathrm{J} / \mathrm{cm}^{2}$ for $3.8 \mathrm{~nm} \mathrm{NCs} ; 242 \mu \mathrm{J} / \mathrm{cm}^{2}$ for $2.6 \mathrm{~nm} \mathrm{NCs}$; and $288 \mu \mathrm{J} / \mathrm{cm}^{2}$ for $2.3 \mathrm{~nm}$ NCs. To ensure that the change in photoluminescence which is observed in these experiments with the IR pump was not due to increased carrier generation, excitation experiments with only IR light were also performed and show no detectable counts in dynamic or static measurements (See Figure S5).

Additional Data and Figures

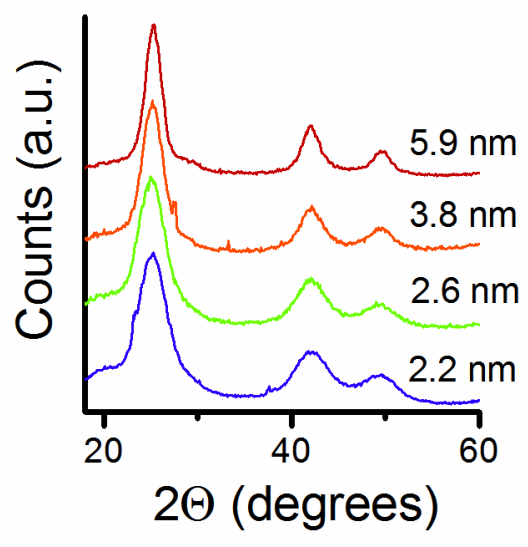

Figure S1. Powder X-ray diffraction measurements of CdSe NCs. 


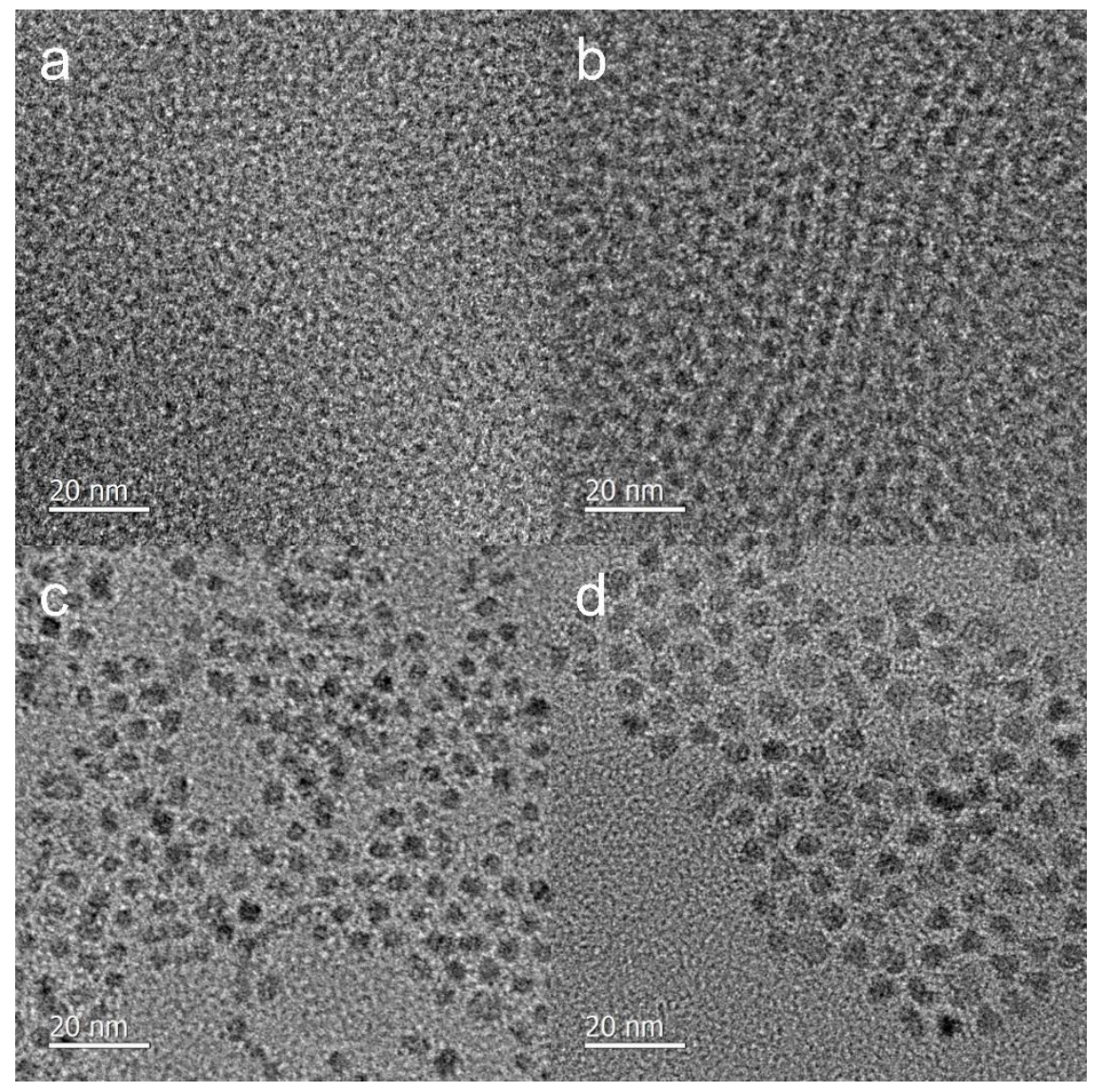

Figure S2. TEM images of (a) $2.3 \mathrm{~nm}$, (b) $2.6 \mathrm{~nm}$, (c) $3.8 \mathrm{~nm}$, and (d) $5.9 \mathrm{~nm}$ zinc blende CdSe NCs.

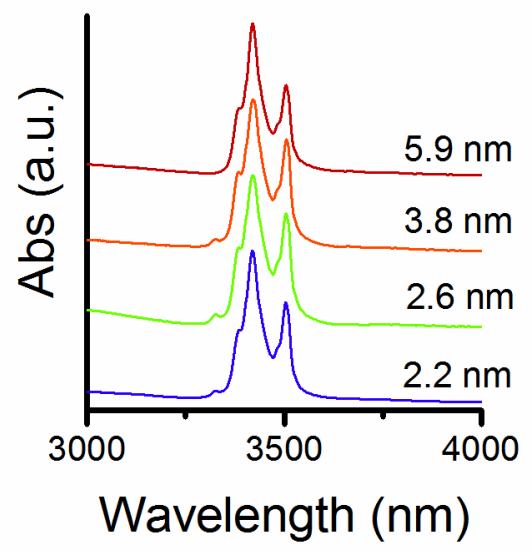

Figure S3. FT-IR data for different sizes of CdSe NCs collected at room temperature on sapphire. 


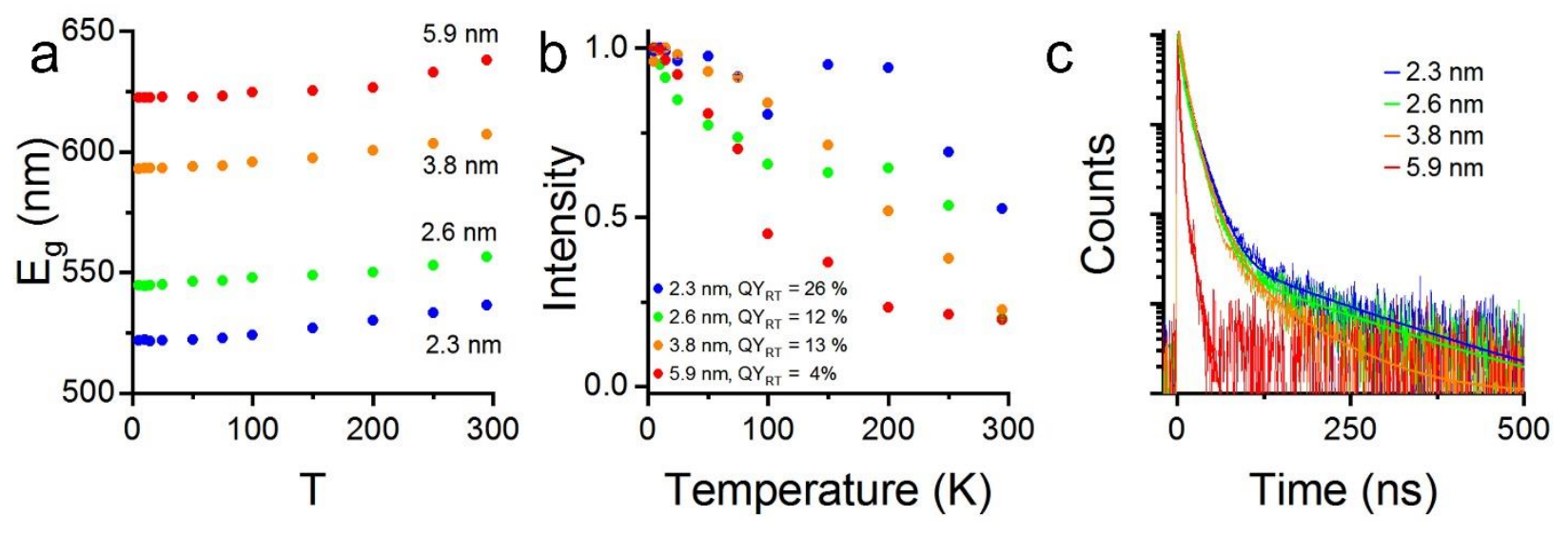

Figure S4. (a) Band gap as a function of temperature, determined from the center emission energy, for the four CdSe QD samples used in this work. The band-gap decreases according to the Varshni relation with increasing temperature. (b) Temperature-dependent intensities of photoluminescence for the samples with the room temperature quantum yields given in the key. Quantum yields at 5 $\mathrm{K}$ are estimated to range from $20-50 \%$. (c) Long-delay time-resolved photoluminescence of the samples. A very long component of emission is observed for $<1 \%$ of the emission. The longest decay component of the samples in (c) was $158 \mathrm{~ns}, 161 \mathrm{~ns}, 82 \mathrm{~ns}$, and $10 \mathrm{~ns}$ for the $2.3 \mathrm{~nm}, 2.6$ $\mathrm{nm}, 3.8 \mathrm{~nm}$, and $5.9 \mathrm{~nm}$ samples, respectively.
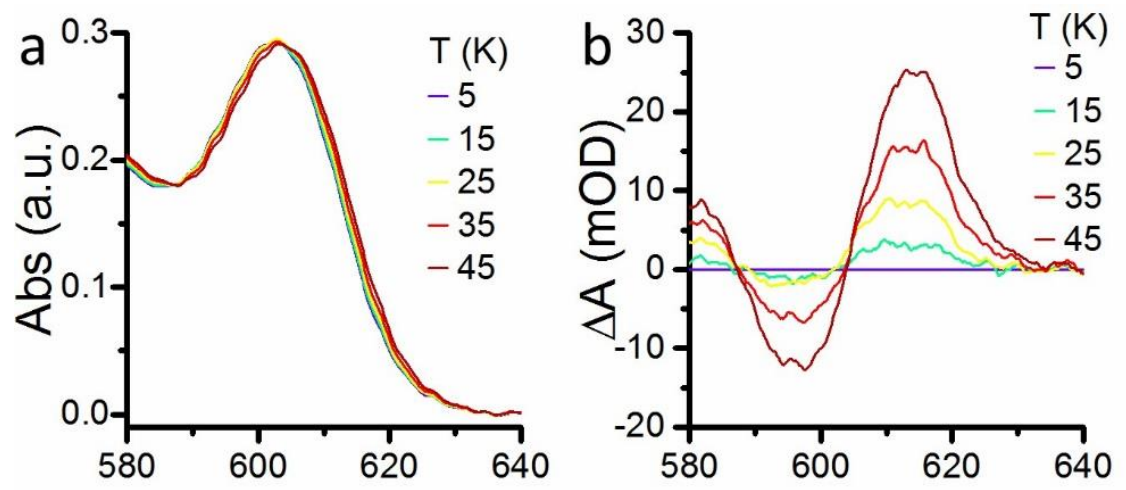

Wavelength $(\mathrm{nm})$
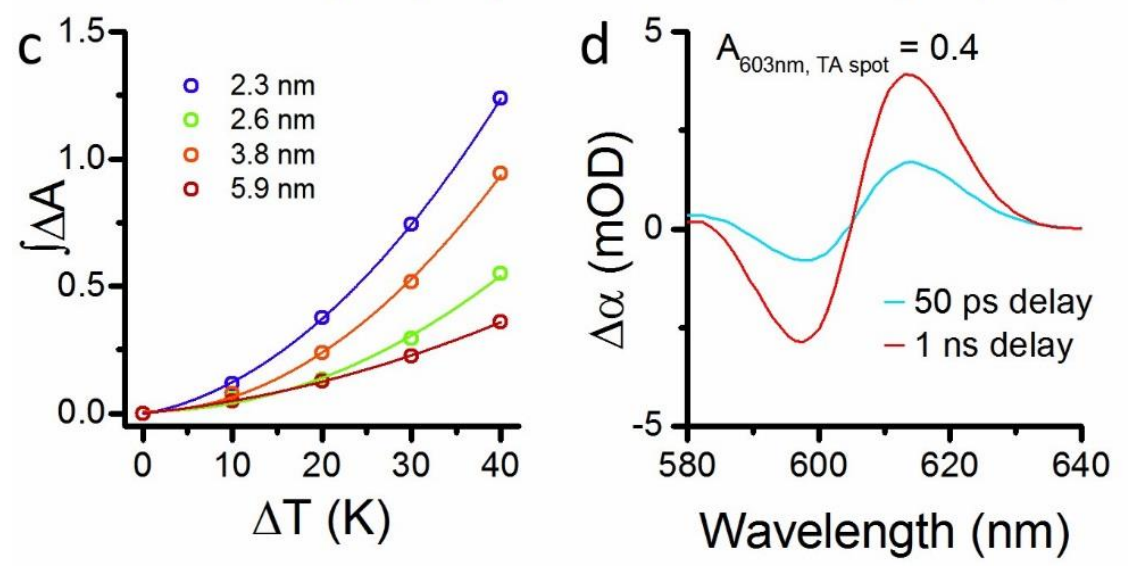

Figure S5. (a) Temperature-dependent absorption measurements of $5.9 \mathrm{~nm}$ CdSe NCs. (b) Static difference spectra of $5.9 \mathrm{~nm} \mathrm{CdSe} \mathrm{NCs} \mathrm{with} \mathrm{respect} \mathrm{to} \mathrm{the} \mathrm{absorption} \mathrm{at} 5 \mathrm{~K}$. (c) Integration of the 
positive lobe of difference spectra for each of the CdSe NCs used in this work. Integration are shown in open circles and solid lines reflect a quadratic fit of the data which was used for calibration of IPEP data. (d) IPEP data for the $5.9 \mathrm{~nm} \mathrm{CdSe} \mathrm{NCs} \mathrm{at} 5 \mathrm{~K}$ for comparison with (b). Critically, the expected $\Delta \alpha$ for a given $\Delta \mathrm{T}$ must be normalized to the total sample absorption at the measured spot, which is somewhat higher than the sample spot used for static absorption spectra in (a).
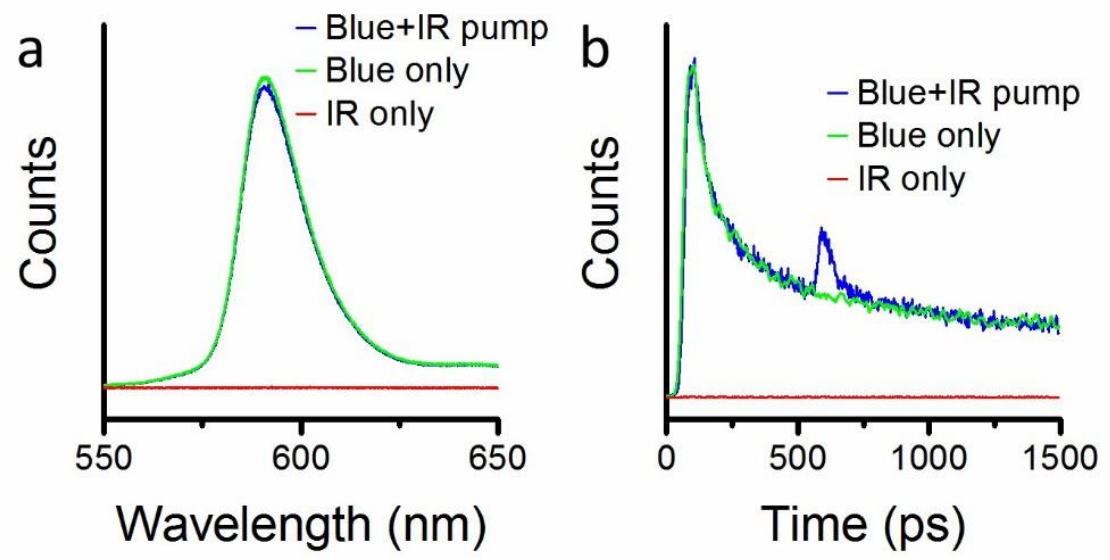

Figure S6. (a) Comparison of time-integrated photoluminescence of $3.8 \mathrm{~nm}$ CdSe NCs at $5 \mathrm{~K}$ under both $400 \mathrm{~nm}$ and $3460 \mathrm{~nm}$ IR excitation (with $500 \mathrm{ps}$ delay), $400 \mathrm{~nm}$ excitation only, and IR excitation only. In practice, any difference which might occur in the time-integrated photoluminescence due to the IR light was found to be smaller than fluctuations in the pump laser intensity which also a dynamic differential photoluminescence. (b) Time-resolved photoluminescence of the same experiment.

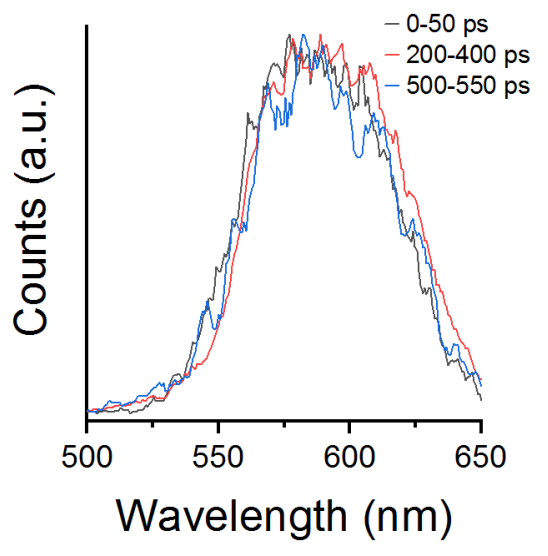

Figure S7. Spectra of $3.8 \mathrm{~nm}$ QDs at different times from streak camera data. The data show a small red-shift after the initial 50 ps; the spectrum after the IR pulse is also slightly blue-shifted. 

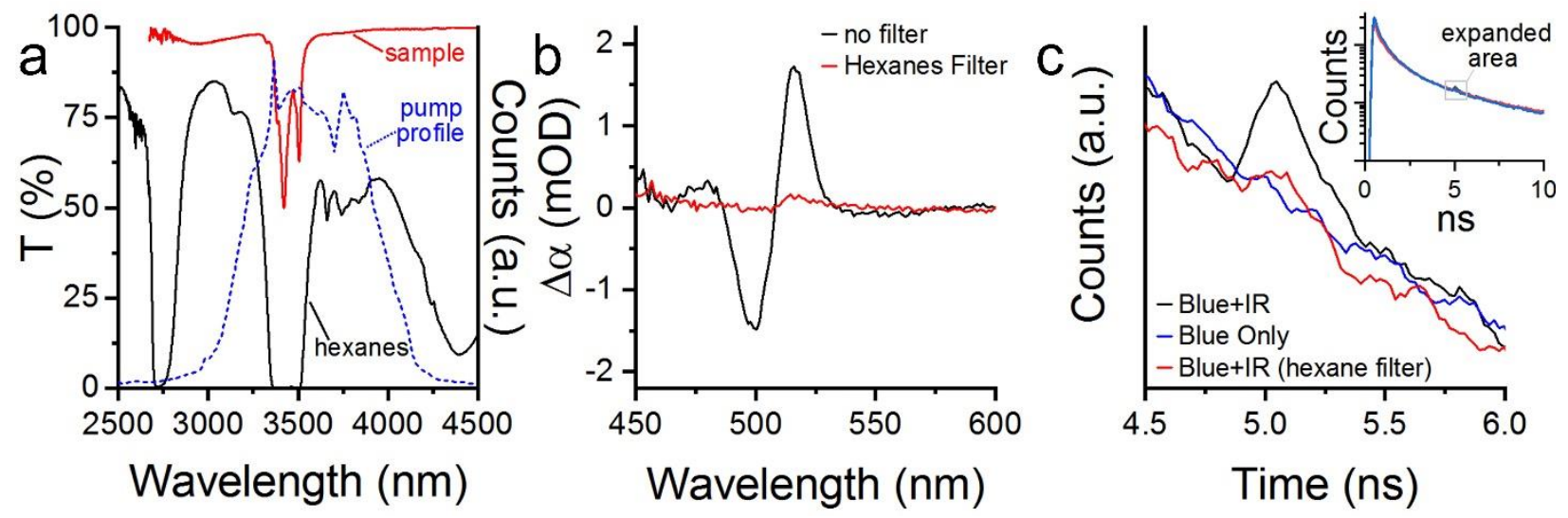

Figure S8. (a) Spectral properties of hexanes dissolved in carbon tetrachloride (in transmission) shown in black. Transmission of sample in mid-IR in red, scaled for clarity. The dashed blue line shows the pump spectrum. Note that the hexanes filter does not fully block the absorption of the ligands, such as from the $\mathrm{sp}^{2} \mathrm{C}$-H stretches. The solid oleate ligands also display somewhat broader absorption band width than the hexanes molecules dissolved in carbon tetrachloride. (b) Comparison of IPEP signal for the same sample at $300 \mathrm{ps}$ delay for the unfiltered probe beam and with the probe beam passing through a cuvette of hexanes dissolved in carbon tetrachloride. (c) Time-resolved photoluminescence of a $3 \mathrm{~nm}$ CdSe quantum dot with blue (400 nm) pump followed by an IR pump at $\sim 5$ ns delay, with only blue pump, and using blue pump followed by IR filtered through hexanes.

\section{REFERENCES}

(1) Yang, Y. A.; Wu, H.; Williams, K. R.; Cao, Y. C. Synthesis of CdSe and CdTe Nanocrystals without Precursor Injection. Angew. Chem., Int. Ed. Engl. 2005, 44 (41), 6712-6715.

(2) Diroll, B. T.; Guo, P.; Schaller, R. D. Unique Optical Properties of Methylammonium Lead Iodide Nanocrystals Below the Bulk Tetragonal-Orthorhombic Phase Transition. Nano Lett. 2018, $18(2), 846-852$.

(3) Diroll, B. T.; Guo, P.; Schaller, R. D. Heat Transfer at Hybrid Interfaces: Interfacial Ligandto-Nanocrystal Heating Monitored with Infrared Pump, Electronic Probe Spectroscopy. Nano Lett. 2018, 18 (12), 7863-7869.

(4) Guo, P.; Mannodi-Kanakkithodi, A.; Gong, J.; Xia, Y.; Stoumpos, C. C.; Cao, D. H.; Diroll, B. T.; Ketterson, J. B.; Wiederrecht, G. P.; Xu, T.; et al. Phonon-Pump Electronic-Probe Study of Methylammonium Lead Iodide Reveals Electronically Decoupled Organic and 
Inorganic Sublattices. 2018, 1-18.

(5) Varshni, Y. P. Temperature Dependence of the Energy Gap in Semiconductors. Physica 1967, 34 (1), 149-154. 